\title{
Temporal Trends in the Loudness of Popular Music over Six Decades
}

\author{
Hourmazd Haghbayan, M.D. ${ }^{1,2,3}{ }^{\oplus}$, Eric A. Coomes, M.D. ${ }^{7}$, and David Curran, B.SC. ${ }^{4}$ \\ 'Department of Medicine, University of Toronto, Toronto, Ontario, Canada; ${ }^{2}$ Division of Cardiology, London Health Sciences Centre, Western \\ University, London, Ontario, Canada; ${ }^{3}$ Department of Social and Preventive Medicine, Université Laval, Québec, Québec, Canada; ${ }^{4}$ OpenJaw \\ Technologies, Dublin, Ireland.
}

J Gen Intern Med 35(1):394-5

DOI: $10.1007 / \mathrm{s} 11606-019-05210-4$

(c) Society of General Internal Medicine 2019

\section{INTRODUCTION}

Repeat recreational exposure to loud music is a key risk factor for noise-induced hearing loss (NIHL) and represents a major public health concern. ${ }^{1}$ The World Health Organization estimates that $50 \%$ of individuals aged 12-35 in developed nations are regularly exposed to unsafe levels of sound from personal music players, with up to 1.1 billion youth worldwide at risk of consequent NIHL. ${ }^{2}$

While prior studies have established the deleterious effects of high music volume expressed in decibels of sound pressure level (dBSPL), ${ }^{3}$ this end-product perceived by the human ear is a composite of several interrelated factors leading to the perception of loudness, including user-controlled volume settings and the intrinsic sound intensity of the sound media itself. In acoustical engineering, the latter may be expressed as decibels relative to full scale (dBFS), an absolute measurement of instantaneous energy which, when all other factors (such as user-defined volume levels) are held equal, is correlated to dBSPL. ${ }^{4}$

Few studies have examined the evolution of the underlying audio engineering of music, a systemic factor which may contribute to NIHL. As industry trends may have progressively accentuated popular music sound intensity over time, a phenomenon generally referred to as the "loudness wars," 5 we undertook a big data analysis of the evolution of such musical sound intensity, as measured in $\mathrm{dBFS}$, to quantitatively assess temporal trends in the loudness of popular music released over the past six decades.

\section{METHODS}

We analyzed the Million Song Dataset, a public dataset of tracks released from 1922 to 2011. The development and

Received May 9, 2019

Accepted July 10, 2019

Published online July 24, 2019 content of this dataset have been previously described. ${ }^{6}$ Briefly, it consists of the metadata of predominantly occidental popular music, with loudness data reported in dBFS. Spanning from the 1920 s to the 2010s, mean (standard deviation [SD]) of song loudness was modelled linearly for each decade that contained $>1000$ songs. Kernel density estimation was used to normalize the frequency distribution of song sound intensity and create ridgeline plots for each decade.

\section{RESULTS}

Combined year and loudness data were available for 515,412 songs released from 1922 to 2011, with all decades from the 1950 s onward containing $n>1000$ songs (total $n=514,579$ ) (panel A). Overall mean (SD) song loudness was -9.7 (4.9) dBFS, with means ranging from -14.4 (5.1) dBFS in the 1950 s to -8.2 (4.3) dBFS in the 2010s. When modelled linearly, mean loudness increased by $1.23 \mathrm{dBFS} / 10$ years $(95 \%$ confidence interval $1.22-$ $1.25)(p<0.001)$ (panel B).

\section{DISCUSSION}

Social exposure to noise from personal music players is a major cause of preventable hearing loss and a growing public health concern. ${ }^{1-3}$ In analyzing a large database of songs spanning several decades, we demonstrate a significant increase in the mean intrinsic sound intensity of popular music (Fig. 1), likely related to progressive changes in audio engineering technology in the transition to digital media. The advent of dynamic range compression in particular, an operation in audio signal processing whereby the differences between peaks and troughs of sound intensity within a song are minimized, results in tracks with consistently higher sound intensity. $^{5}$

Although our study demonstrates an ongoing, progressive accentuation of the intrinsic sound intensity of popular music over time, a limitation is that the subsequent user-level response in volume adjustment cannot be accounted for. However, higher volume levels and longer durations of exposure are well-established user-level risk factors for $\mathrm{NIHL}^{1,3}$ and 

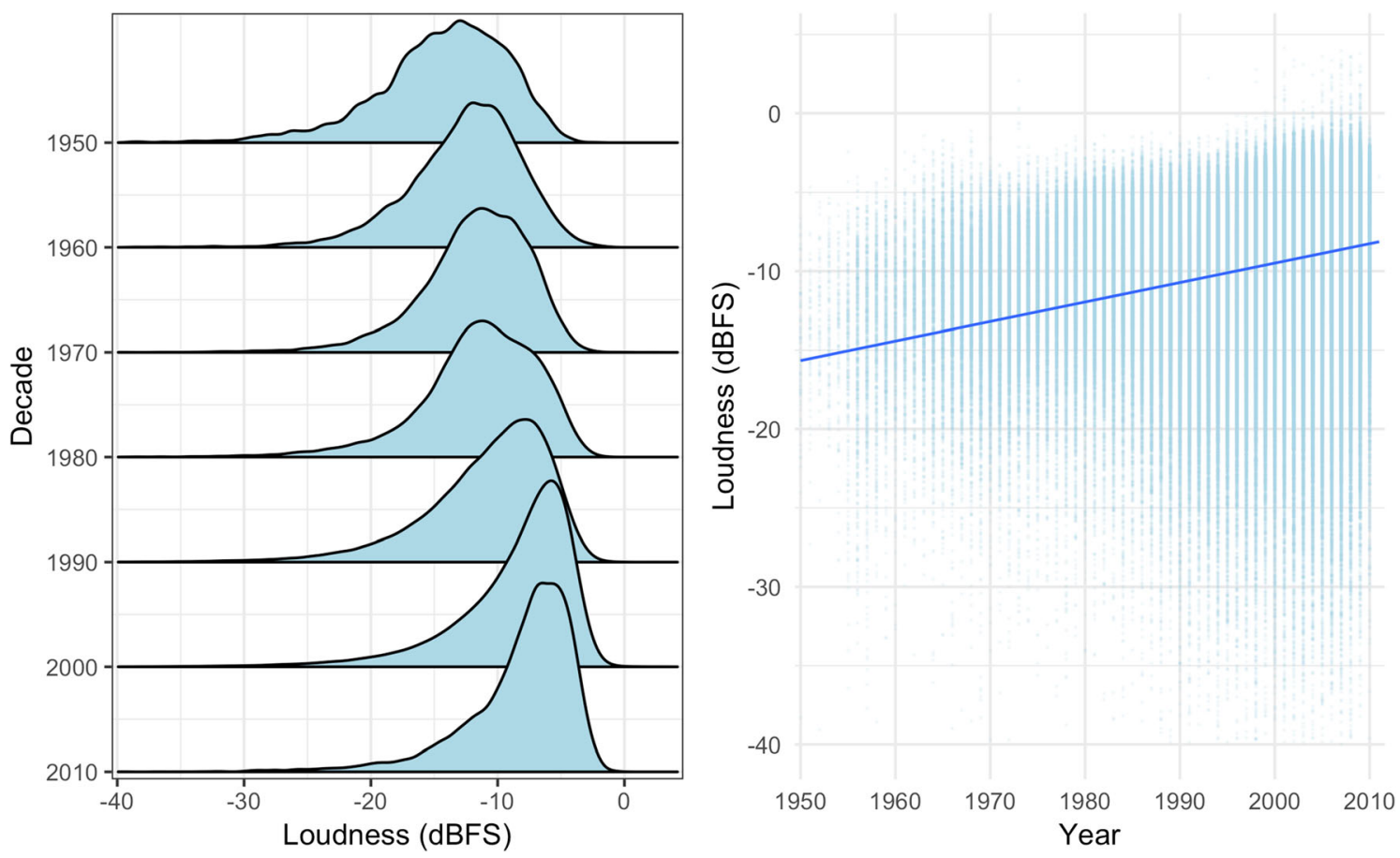

Figure 1 Temporal trends in the loudness of songs in decibels relative to full scale (dBFS) by decade, 1950s to 2010s. a Ridgeline plots of popular music song loudness by decade; kernel density estimation was used to normalize the frequency distribution of loudness in decibels relative to full scale (dBFS) for each decade from 1950s to 2010s. The maximum height of each curve represents the most common (modal) loudness in dBFS occurring in the associated decade. b Popular music song loudness as a function of time, modelled linearly.

dynamic range compression may exacerbate these phenomena as there are fewer quiet troughs providing "rest periods" within tracks. ${ }^{5}$ Additionally, this imposes increased onus on the part of the consumer to engage in frequent manual volume adjustments between tracks of varying compression to prevent deleterious sound levels. Such trends in popular music sound intensity therefore represent a potentially preventable, but music industry-level, risk factor for NIHL which should be the target of future research.

Corresponding Author: Hourmazd Haghbayan, M.D.; Division of Cardiology, London Health Sciences Centre Western University, London, Ontario, Canada (e-mail: hourmazd.haghbayan@mail. utoronto.ca).

\section{Compliance with Ethical Standards:}

Conflict of Interest: The authors declare that they do not have a conflict of interest.
Publisher's Note: Springer Nature remains neutral with regard to jurisdictional claims in published maps and institutional affiliations.

\section{REFERENCES}

1. Basner M, Babisch W, Davis A, et al. Auditory and Non-Auditory Effects of Noise on Health. Lancet. 2014;383(9925): 1325-1332.

2. World Health Organization. Hearing Loss Due to Recreational Exposure to Loud Sounds: A Review. 2015.

3. Scientific Committee on Emerging and Newly Identified Health Risks (SCENIHR). Potential Health Risks of Exposure to Noise from Personal Music Players and Mobile Phones Including a Music Playing Function. European Commission. 2008. http://ec.europa.eu/health/ph_risk/committees/04_scenihr/docs/scenihr_o_018.pdf.

4. Biamp Systems. Gain Structure: Input and Output Levels. Cornerstone. 2018. https://support.biamp.com/General/Audio/Gain_structure\%3A_ input_and_output_levels. Accessed December 29, 2018.

5. Vickers E. The Loudness War: Background, Speculation, and Recommendations. Audio Eng Soc Conv. 2010;129:1-27.

6. Bertin-Mahieux T, Ellis DPW, Whitman B, Lamere P. The Million Song Dataset Challenge. Proc 12th Int Soc Music Inf Retr Conf. 2011:591-596. 